\title{
Vaccine safety in an era of novel vaccines: a proposed research agenda
}

Gregory A. Poland (10) ${ }^{凶}$ and Richard B. Kennedy (D)

Vaccines have outstanding efficacy and safety records, and the evolving science of vaccines is enabling us to better understand their mechanisms of action as well as the pathways that drive vaccine-related adverse events. This understanding is particularly crucial as novel vaccine antigens, platforms and adjuvants are increasingly being used.

The past decade, and particularly the past few years, has seen a rapid acceleration in novel vaccine development, owing to public health emergencies (such as COVID-19 and Ebola), public health urgencies (cholera, meningococcal disease and others) and public health necessities (pneumococcal disease, influenza, hepatitis B and others), as well as improvements to existing vaccines. These developments have been made possible and informed by advances in basic biology and vaccine science, including new vaccine antigens, new vaccine platforms (mRNA and adenovirus vectored) and new vaccine adjuvants.

By virtue of their approval, these vaccines are effective and safe. But how safe? And by what measures? In the US, a robust, redundant and synergistic vaccine safety network exists, involving programmes such as the Vaccine Adverse Event Reporting System (VAERS). These programmes have enabled, in near real time, the identification of very rare side effects associated with mRNA and adenovirus-vectored COVID-19 vaccines, such as vaccine-induced immune thrombotic thrombocytopenia (VITT), myocarditis, pericarditis and Guillain-Barré syndrome. Notably, these programmes are well suited to identifying singular major adverse events that have well-defined phenotypic definitions and occur with a strong temporal component. By contrast, these systems are not well adapted to recognizing adverse events without strong phenotypic markers or temporal associations, or those that may be subclinical.

In the context of a public health emergency, there must also necessarily be a balance between rapid deployment of a life-saving vaccine and investigation of less severe adverse events. For example, there have been thousands of reports of the onset and/or worsening of tinnitus in recipients of COVID-19 mRNA vaccines through VAERS and other mechanisms. So far, to our knowledge, the CDC Vaccine Safety Office has not formally explored this potential adverse event. Tinnitus is a common problem, with a definable background population rate, that has important 'quality of life' implications. But, it is a condition with no observable or measurable phenotypic markers, other than associated hearing loss.
How can adverse events be identified and quantified if they cannot be 'measured' by standardized diagnostic tests? Similarly, we have only nascent understandings of the theoretical effects of vaccines, such as effects on epigenetics, trained immunity, antigenic imprinting and longer-term mutational pressure. Significant research is necessary to understand more comprehensively the possibility of novel adverse effects of novel vaccines.

\section{Known vaccine-related adverse events}

Vaccines have known side effects, both local and systemic, that may be caused by various mechanisms. Vaccine components are tested for toxicity during preclinical development, but it is possible that toxic reactions may only occur in specific situations or in rare population groups and therefore will not be apparent until larger clinical trials or after widespread use in the general population. Inappropriate immune reactions can also drive vaccine-induced side effects through several mechanisms: overstimulation of innate immunity; cross-reactive adaptive immune responses to host epitopes; and the generation of autoimmune responses.

A crucial property of vaccines is the ability to elicit a sufficiently strong innate immune response to enhance antigen presentation and costimulatory signals necessary for robust cellular immune activation. However, these innate immune responses are also responsible for most of the common, transient side effects after vaccination, such as injection site pain, fever and myalgia. More serious events, including allergic reactions and anaphylaxis, can occur but are fortunately rare. Vaccine developers must therefore find the right balance to achieve the minimum reactogenicity necessary for optimal immunogenicity and efficacy.

Cross-reactive immune responses (involving either $\mathrm{T}$ cells or B cells) are another key consideration in vaccine design. Cross-reactivity is responsible for the protective efficacy of vaccinia virus vaccine against variola virus (smallpox) as well as of the BCG vaccine (containing Mycobacterium bovis) against Mycobacterium tuberculosis ${ }^{1}$. Also, immune responses to pneumococcal, 
meningococcal, rotavirus, human papillomavirus and other vaccines provide broader protection owing to cross-reactivity against pathogen strains not included in the vaccine $e^{2-4}$. However, cross-reactivity can on rare occasions target host proteins and lead to immunopathology. One example of this is the development of narcolepsy in a small number of recipients of Pandemrix, the AS03-adjuvanted influenza $\mathrm{A} / \mathrm{H} 1 \mathrm{~N} 1$ vaccine, associated with the HLA-DQB1 ${ }^{*} 06: 02$ haplotype ${ }^{5}$. Sequence similarity between the influenza nucleoprotein and the receptor for hypocretin (a neurotransmitter that regulates sleep) was proposed as a potential mechanism. Antibodies from affected individuals cross-react with both proteins, and transfer of these antibodies into mice recapitulated the narcoleptic condition ${ }^{6,7}$. A more contemporary example is the development of VITT in a small number of recipients of adenovirus-vectored COVID-19 vaccine. Multiple hypotheses, including cross-reactivity between antibodies to SARS-CoV-2 and platelet factor 4 have been proposed, but no clear mechanism has been identified so far'.

Excessive or chronic inflammation, which could result from infection or vaccination, has also been implicated in promoting the development of autoimmune responses. The data supporting a link between infection and the development of autoimmune disorders are relatively strong and have been reviewed elsewhere ${ }^{9}$. Multiple large, observational and epidemiological studies have been carried out to examine the link between vaccines and allergic or autoimmune diseases ${ }^{10}$. Although these studies have limitations, there is a consistent lack of evidence linking vaccines to autoimmune disease. Nevertheless, the possibility deserves continued attention as we develop new vaccines.

\section{Proposed future research agenda}

Safety is a primary consideration in vaccine development, and because vaccines are typically given to healthy individuals to protect against a possible future disease, the bar for safety is necessarily much higher than for other medical interventions. Public interest in, and scrutiny of, vaccine safety has also increased considerably. We believe that vaccine safety is an area in which continued research will provide valuable information to support public heath, which will require increased funding and infrastructure support for basic, translational and clinical research.

First, increased basic research into the molecular and immunological mechanisms elicited by vaccines will provide crucial information regarding the pathways that drive adverse events. This knowledge could potentially enable such problems to be avoided in future vaccines. For example, research into the mechanisms of VITT could be used to modify future iterations of adenovirus-vectored COVID-19 vaccines.

Second, continued research in the area of adjuvants will expand our ability to fine-tune innate and adaptive immune responses to optimize protection against different pathogens, while minimizing potentially pathological reactogenicity. For example, ongoing research looks to create type 1-biased or type 2-biased immune responses or to specifically enhance mucosal immunity.
Third, increased use of systems biology approaches will be needed to fully characterize, assess and understand the complex interactions between cells, tissues and molecules that constitute immune responses, both protective and aberrant or pathological.

Fourth, we should invest in heightened surveillance systems during clinical trials and post-marketing. This should include a better understanding of the background rates of potential complications, as well as syndromic surveillance, recognizing the importance of identifying collections of adverse events.

\section{Conclusions}

Robust and trustworthy safety surveillance systems that serve the public agenda are essential for public trust and acceptance of vaccines. Furthermore, the identification of safety issues advances the science by allowing for the identification of mechanisms involved in such events and thereby the reverse engineering of new vaccine candidates to avoid activating such mechanisms. Surveillance systems can also enhance our understanding of who will experience vaccine-related adverse events and under what conditions. Can safety issues be predicted at the individual level in the future? How should this inform new vaccine development and vaccine public health policy? Ultimately, we need to accumulate the data required to place risk and benefit in context; no vaccine will ever be completely free of adverse effects the question is whether the frequency of adverse effects is countered by correspondingly sufficient benefit?

1. Vojtek, I., Buchy, P., Doherty, T. M. \& Hoet, B. Would immunization be the same without cross-reactivity? Vaccine 37, 539-549 (2019). 2. Wheeler, C. M. et al. Cross-protective efficacy of HPV-16/18 AS04adjuvanted vaccine against cervical infection and precancer caused by non-vaccine oncogenic HPV types: 4-year end-of-study analysis of the randomised, double-blind PATRICIA trial. Lancet Oncol. 13 , 100-110 (2012).

3. Cates, J. E. et al. Do rotavirus strains affect vaccine effectiveness? A systematic review and meta-analysis. Pediatr. Infect. Dis. J. 40, 1135-1143 (2021).

4. Watson, P. S., Novy, P. L. \& Friedland, L. R. Potential benefits of using a multicomponent vaccine for prevention of serogroup $B$ meningococcal disease. Int. J. Infect. Dis. 85, 22-27 (2019).

5. Partinen, M. et al. Narcolepsy as an autoimmune disease: the role of $\mathrm{H} 1 \mathrm{~N} 1$ infection and vaccination. Lancet Neurol. 13, 600-613 (2014).

6. Ahmed, S. S. et al. Antibodies to influenza nucleoprotein cross-react with human hypocretin receptor 2. Sci. Transl Med. 7, 294 ra 105 (2015).

7. Katzav, A. et al. Passive transfer of narcolepsy: anti-TRIB2 autoantibody positive patient $\lg \mathrm{G}$ causes hypothalamic orexin neuron loss and sleep attacks in mice. J. Autoimmun. 45, 24-30 (2013).

8. Bilotta, C. et al. COVID-19 Vaccine-related thrombosis: a systematic review and exploratory analysis. Front. Immunol. 12, 729251 (2021).

9. Wraith, D. C., Goldman, M. \& Lambert, P. H. Vaccination and autoimmune disease: what is the evidence? Lancet 362 , 1659-1666 (2003).

10. Offit, P. A. \& Hackett, C. J. Addressing parents' concerns: do vaccines cause allergic or autoimmune diseases? Pediatrics 111 653-659 (2003).

Competing interests

G.A.P. offers consultative advice on vaccine and antiviral development to AstraZeneca, Pfizer, Medicago, Emergent Biosolutions, Johnson\&Johnson/ Janssen, Novavax, Moderna, Merck Research Laboratories, Glaxo SmithKline, Sanofi Pasteur, Eli Lilly and Company, Exelixis, Genevant, Regeneron Pharmaceuticals, Syneos Health and Vyriad. G.A.P. has received grant funding and royalties from ICW Healthcare Ventures for preclinical studies on a peptide-based COVID-19 vaccine for which he is an inventor. These activities are conducted in compliance with Mayo Clinic Conflict of Interest policies. R.B.K. has received royalties from ICW Healthcare Ventures for licensing of peptide vaccine development patents and intellectual property owned by Mayo Clinic of which he is an inventor or co-inventor. R.B.K. has consulted with Merck Research Laboratories on measles vaccines and with Sanofi Pasteur and Outcomes Insights on influenza vaccines. 